\title{
COMPORTAMIENTO DENSO-GRANULOMÉTRICO Y QUÍMICO DE LAS COLAS DE LA TECNOLOGIA CARON EN MOA
}

\author{
Y.C. CALA, A.P. RODRÍGUEZ, P.E.B. MAZAR, O.L. BREFFE \\ Instituto Superior Minero Metalúrgico de Moa \\ ycorreac@ismm.edu.cu
}

Artigo submetido em 07/09/2018 e aceito em 28/10/2019

DOI: $10.15628 /$ holos.2019.7706

\section{RESUMEN}

La tecnología CAROM de Moa, Cuba, vierte cada año millones de toneladas de colas al medio ambiente con alta concentración de especies metálicas valiosas que pueden ser recuperadas, por esta razón el objetivo de este trabajo, es determinar el comportamiento densogranulométrico y químico de las colas para su separación. Para ello se utilizó el método de elutriación para separar las partículas teniendo como base la combinación de las propiedades granulometría y densidad. La composición química se determinó a través de la técnica de Espectroscopía de Fluorescencia de rayos $X$. Se obtuvo que, en el reboso, en las fracciones más finas se pueden recuperar el hierro y aluminio, a la mayor y menor densidad respectivamente. En la fracción gruesa a la menor densidad se recupera el níquel, magnesio y silicio. En las fracciones más finas y a menor densidad de las arenas se recupera el manganeso, mientras a la mayor densidad lo hacen el cobalto y cromo.

PALABRAS-CLAVE: Colas, Densidad, Elutriación, Granulometría.

\section{DENSE-GRANULOMETRIC AND CHEMICAL BEHAVIOR OF THE TAILINGS FROM CARON TECHNOLOGY IN MOA}

\begin{abstract}
The CAROM technology of Moa, Cuba, pours millions of tons of tailings into the environment every year with a high concentration of valuable metallic species that can be recovered, for this reason the objective of this work is to determine the dense-granulometric and chemical behavior of the tailings for their separation. For this, the elutriation method was used to separate the particles based on the combination of granulometry and density properties. The chemical composition was determined through the technique of $\mathrm{X}$-ray fluorescence spectroscopy.
\end{abstract}

It was obtained that, in the overflow, in the finest fractions iron and aluminum can be recovered, at the highest and lowest density respectively. In the coarse fraction at the lowest density, nickel, magnesium and silicon are recovered. In the finer fractions and lower density of the sands, manganese is recovered, while at the highest density cobalt and chromium are recovered.

KEYWORDS: Tailings, Density, Elutriation, Granulometry. 


\section{INTRODUCCIÓN}

La elutriación es una de las técnicas de separación gravimétrica más usada en la clasificación por tamaño y densidad de partículas minerales. Ésta puede llevarse a cabo vía húmeda o seca, dependiendo del tipo de fluido (líquido o gas), siendo el método húmedo la técnica más sencilla y de menor complejidad. Tal procedimiento se basa en la diferencia de la velocidad de sedimentación adquiridas por las partículas sólidas al viajar a través de un fluido en movimiento (Coulson \& Richardson, 1979).

La generación de colas por las industrias de la tecnología caron en el territorio holguinero por más de 50 años, con alto contenido de hierro y otros metales pesados, constituye uno de los principales problemas de mayor impacto ambiental; por ser una fuente de contaminación que afecta la erosión de los suelos, a la flora y fauna terrestre, a las zonas costeras aledañas a los depósitos y a las aguas superficiales y subterráneas, como consecuencia del alto régimen de precipitación en la región. A lo anterior se suma molestia y contaminación atmosférica ocasionada en los asentamientos poblacionales cercanos en época de sequía.

Diferentes investigadores del territorio han realizado estudios referentes a las colas de la tecnología carbonato amoniacal de Moa y Nicaro, entre los que se destacan (Ponce, 1979; Ponce \& Altarriba, 1986; Herrera, 1961; Ariza et al. 2004; Palmero, 2011; Del Toro, 2003 y Loyola et al. 2014). En estas investigaciones llegaron a la conclusión que las colas son un residual con alto contenido de especies metálicas muy valiosas, de granulometría fina y tienen como fase mineralógica principal la maghemita y magnetita.

Las primeras referencias de trabajos realizados con el objetivo de aprovechar las colas en forma de concentrado de hierro aparecen en 1952 por la National Lead Company; en las investigaciones se realiza una nodulación de las colas y se calcinan a 1173,16 K, los nódulos se mezclan con cisco de coque y carbonato de calcio, se calientan hasta $1423,16 \mathrm{~K}$, se enfrían, trituran y se realiza la separación magnética seca, donde se obtiene un producto magnético con $71,2 \%$ de hierro y recuperación del $83,4 \%$. Similar procedimiento se realiza con mezcla 1:1 de colas y mineral de hierro de baja ley para obtener un producto magnético con 91,1 \% de hierrototal y recuperación del 73,9 \% (Kowalski, 1953). Pero tiene como inconveniente que es un proceso altamente consumidor de energía.

Entre los años 1979 y 1981 se realizan, en el Instituto Mejanobr de Leningrado y el SIME en Cuba, pruebas de separación magnética de la mezcla de colas tomadas del depósito y la producción corriente en la planta de Nicaro, con el objetivo de determinar los índices de beneficio y luego producir concentrado para las pruebas de sinterización. Se aplica la filtración y sinterización a los concentrados obtenidos. Se obtuvo concentrados entre 55,9 y $57,0 \%$ de hierro y entre 1,8 y $2,0 \%$ de cromita. Lo obtenido fue adecuado para las pruebas de sinterización; pero, poseen la desventaja de la presencia de hidrocarburos en la cola tomada, los cuales ejercen influencia negativa en la estabilidad de los procesos de separación y filtración, así como en los índices tecnológicos correspondientes (Resumen Taller de colas, 2005).

Ponce (1979) estudió la mineralogía y composición sustancial de las colas de Nicaro. Para ello emplea difracción de rayos $X$, microscopía óptica y ensayos térmicos; donde determinó las fases mineralógicas siguientes: magnetita, hematita, cromita, forsterita y andalucita. El mismo 
investigador en 1986 estudió la granulometría de residuos sólidos de este proceso en el depósito de Moa. En su trabajo no se indica una tecnología para el tratamiento de las colas.

Peña (1986) estudió la granulometría de las colas de Nicaro para la obtención de un concentrado más rico en hierro; analizando diferentes métodos de estudio, como la variación en el esquema de separación magnética y utilización de la flotación. El autor concluye que la separación magnética es el método más apropiado para el beneficio de las colas; mientras que, por el método de flotación no se pudo obtener un concentrado final con alto contenido de hierro. Se consideró realizar un análisis mineralógico para el estudio de las diferentes fases del hierro y obtener mejores resultados en la concentración.

Rojas (2003) realizó una caracterización mineralógica de las colas de la tecnología CARON donde encuentra la existencia de las fases cromoespinelas: $\mathrm{Mg}$ cromita $(\mathrm{Mg}, \mathrm{Fe})\left(\mathrm{Cr}_{2} \mathrm{O}_{3}\right)$, donathita $\left((\mathrm{Fe}, \mathrm{Mg})(\mathrm{Cr}, \mathrm{Fe})_{2} \mathrm{O}_{4}\right)$ y trevorita $\left(\mathrm{Ni}, \mathrm{Fe}_{2} \mathrm{O}_{4}\right)$ y como fases mineralógicas secundarias Cuarzo $\left(\mathrm{SiO}_{2}\right)$ y silicatos de hierro y magnesio de la serie del Olivino, Forsterita- $\mathrm{Fe}(\mathrm{Mg}, \mathrm{Fe})_{2}\left(\mathrm{SiO}_{4}\right)$.

Rojas \& Turro (2003) analizaron la composición mineralógica, química y granulométrica de las colas del proceso CARON de Moa, se tomó muestras de las plantas de lixiviación y recuperación de amoniaco, llegando a la conclusión que las colas obtenidas presentaron tres grupos granulométricos principales: uno predominante, constituído por la fracción menor de 0,044 mm; otro que agrupa las clases granulométricas $(+0,175 \mathrm{~mm}),(-0,147+0,074 \mathrm{~mm})$ y $(-0,074+0,044 \mathrm{~mm})$ y la clase granulométrica gruesa $(0,175+0,147 \mathrm{~mm})$. Los óxidos de hierro maghemita y/o magnetita constituyeron las fases mineralógicas principales y, además, no existió diferencia significativa en la composición química de las colas en ambas plantas; pero no aportaron una tecnología para el tratamiento de las colas.

Medrano (2007) efectuó un estudio físico y químico a las colas de la empresa "Comandante Ernesto Che Guevara". En el análisis químico se obtuvo resultados muy parecidos a los realizados por Col \& García (1984) a las colas de Nicaro. El análisis picnométrico reflejó que la cola posee 42,5 $\%$ de poros y humedad molecular capilar de $42,4 \%$. El contenido del hierro aumentó con la disminución del tamaño de las partículas; mientras que, para el cromo el mayor contenido estuvo entre 0,125 y 0,030 $\mathrm{mm}$, el contenido de óxido de aluminio presentó una regularidad bien definida alrededor del $3 \%$. A pesar de los buenos resultados se considera que se debe realizar análisis mineralógico de los elementos para analizar su comportamiento.

Loyola et al. (2014) evaluaron el comportamiento, ante la acción de la intensidad del campo magnético de colas del proceso Caron de Moa, con vistas a la recuperación de metales útiles contenidos en estos residuos industriales. La investigación se realizó a escala de laboratorio para tres clases granulométricas obtenidas por vía húmeda y, posteriormente, sometidas a separación en fracciones magnéticas y no magnéticas. Se encontró que el níquel y la sílice, mayormente, se concentran en la fracción no magnética de la clase de mayor tamaño. En la fracción gruesa magnética se obtuvo hasta $0,1 \%$ de cobalto, valor exigido por la tecnología Caron. El hierro, aunque se logró concentrar en un 50 \% en la fracción fina magnética, no cumple con las expectativas tecnológicas para su uso en la obtención de aceros.

Sobre la base de los resultados obtenidos en las investigaciones analizadas; se puede concluir que el procedimiento más utilizado para la concentración del hierro y cromo, en las colas de la 
tecnología carbonato amoniacal, es la separación magnética húmeda a baja intensidad de campo magnético; mediante el cual se logra alcanzar concentrados entre 50 y $60 \%$ de hierro.

Todo lo anterior trae como consecuencia que se busque nuevas alternativas tecnológicas que cuenten con infraestructura especializada, en base a los procesos convencionales. Así se puede lograr un tratamiento sostenible de los residuos, de manera tal que permitan un tratamiento integral y posibiliten la sostenibilidad del desarrollo minero de la región. Por esta razón el objetivo de este trabajo es determinar el comportamiento denso granulométrico y químico de las colas para su separación.

\section{MÉTODOS Y MATERIALES}

Las colas son un material fino de color negro y constituye el desecho de la planta de Lixiviación y Lavado, las mismas contienen una elevada cantidad de metales que si se logran recuperar se resuelve un gran problema a la sociedad desde el punto de vista ambiental, económico y social. En ella se desconoce el comportamento de las especies metálicas teniendo en cuenta la combinación de la granulometria y densidad. En tal sentido es tratada por una serie de técnicas experimentales y analíticas que se describen a continuación.

\subsection{Diseño de experimentos}

El diseño experimental aplicado fue el Factorial Multinivel, el cual cuenta con la combinación de las variables de entrada densidad y granulometría. Las variables de salida fueron la masa mineral y la composición química. Los parámetros constantes fueron, $\mathrm{pH}$; contenido de sólido y temperatura. Este modelo consiste en un diseño del tipo:

$$
N=p \cdot q
$$

Donde:

N. Número de experimentos

$p$. Factor fracciones granulométricas (4)

q. Factor nivel de densidad (3).

Por tanto, la cantidad de experimentos es 12.

Las combinaciones para llevar a cabo los experimentos fueron cuatro fracciones granulométricas 0,$02 ; 0,04 ; 0,06$ y $0,08 \mathrm{~mm}$ combinadas con tres densidades de 2800; 3200 y 3600 $\mathrm{kg} / \mathrm{m}^{3}$. Cada fracción granulométrica se combinó con las tres densidades. La densidad de las colas se determinó mediante picnometría.

Para estimar si existe o no diferencia significativa entre cada uno de los elementos en los productos reboso y arenas se empleó el procedimiento de diferencia mínima significativa (LSD) o método de Fisher, por medio del cual se comprueba la significación estadística de cada efecto comparando la media al cuadrado contra una estimación del error experimental. Los efectos que tienen los p-valores inferiores a 0,05, indican que son significativamente diferentes al 95,0\% de nivel de confianza. 


\subsection{Metodología experimental}

La metodología seguida a partir del método de elutriación, se basa en las experiencias de trabajo precedente para distintos materiales (Goodell, 1980; Peña, 1982 y García 1988). El estudio experimental se lleva a cabo mediante el esquema de la figura 1.

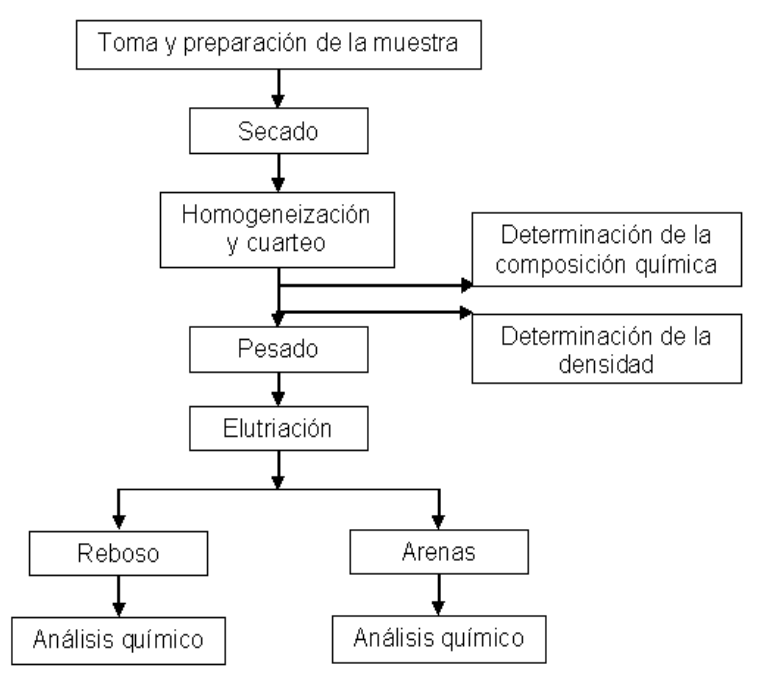

Figura 1: Esquema para el tratamiento de las colas por elutriación.

\subsection{Flujo para el tratamiento de las colas}

En el estudio se tomó una muestra directamente de la presa de colas, mediante el método de redes y puntos (Mitrofanov, 1982), el material, con alto contenido de humedad, se secó y se homogeneizó con el método de cuarteo y anillo. Seguidamente, se pesó tres muestras de 0,250 kg y se determinó el gasto de agua que debe pasar para arrastrar un tamaño de partícula determinado mediante la ley de Stoke y la fórmula del gasto volumétrico, quedando como sigue:

$V=\frac{g \cdot d^{2} \cdot\left(\rho_{p}-\rho_{a}\right)}{18 \cdot \mu} \cdot F ;\left(\mathrm{m}^{3} / \mathrm{s}\right)$

donde:

$V$ : gasto volumétrico de agua, $\frac{m^{3}}{s}$;

$d$ : diámetro de partículas, $m$;

$\rho_{p}$ У $\rho_{a}$ : densidad de partículas sólidas y del agua, $\mathrm{kg} / \mathrm{m}^{3}$;

$\mu$ : coeficiente de viscosidad dinámica del agua, $\mathrm{Pa} \cdot \mathrm{s}$;

$F$ : superficie por donde se mueve el flujo, $\mathrm{m}^{2}$;

g: aceleración de la gravedad, $\mathrm{m} / \mathrm{s}^{2}$;

En el proceso de elutriación se obtiene dos produtos, reboso $(R)$ y arenas $(A)$. 
Para realizar los experimentos, se comenzó con la combinación $0,02 \mathrm{~mm}$ y $2800 \mathrm{~kg} / \mathrm{m}^{3}$, el producto obtenido (arenas) se somete al próximo experimento con la misma granulometría, pero a la densidad de $3200 \mathrm{~kg} / \mathrm{m}^{3}$, el último experimento relacionado con esta granulometría se realizó con con la densidad de $3600 \mathrm{~kg} / \mathrm{m}^{3}$. El proceso de elutriación se realiza con el producto arenas. El producto obtenido de la combinación $0,02 \mathrm{~mm}$ y $3600 \mathrm{~kg} / \mathrm{m}^{3}$ se somete a la operación antes descrita pero con la fracción $0,04 \mathrm{~mm}$, este tamaño de partícula se combina con las tres densidades. Terminada esta combinación se realizó el mismo procedimiento, pero con los tamaños de partículas de 0,06 y $0,08 \mathrm{~mm}$ respectivamente.

En la figura 2 se muestra la instalación que se empleó para realizar los experimentos. Consta de un tanque que suministra agua al proceso; a la salida del tanque se conecta una válvula, la que debe estar abierta para dar paso del agua a la bomba, a la salida de la misma se acopla otra válvula que tiene como fin regular el flujo de agua que se suministra al elutriador.

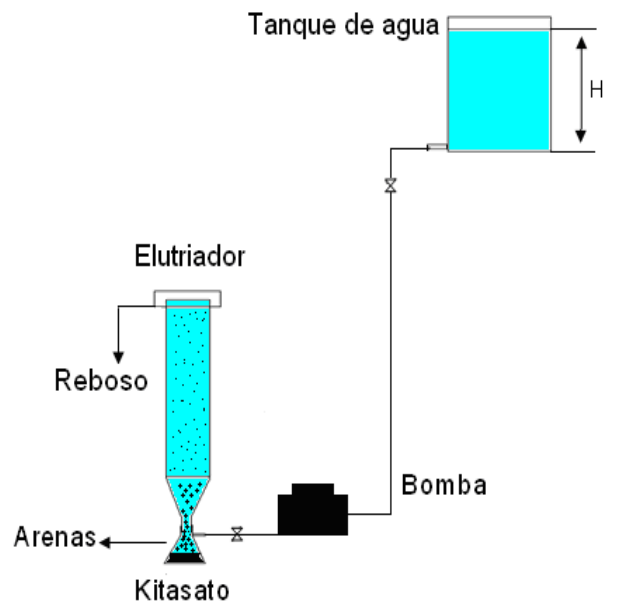

Figura 2: Instalación para la realización de los experimentos.

En la parte inferior del equipo se encuentra instalado un kitasato, en el cual se recoge el producto arenas; en la parte superior se conecta un recipiente que recibe el producto reboso. Los productos se obtienen en forma de pulpa; por tanto, se dejan sedimentar por espacio de 24 horas aproximadamente, después se decantan y se secan para obtener el material sólido y continuar con el flujo tecnológico propuesto.

Los dos produtos, después que se secan, se sometieron a análisis químico mediante Espectroscopia de Fluorescencia de Rayos X, para determinar las especies metálicas existentes en ellos, así como la cantidad en porcentaje de los mismos en cada combinación escogida.

\section{RESULTADOS Y DISCUSIÓN}

Luego de explicar las técnicas experimentales y analíticas a emplear se procede a la interpretación de los principales resultados.

\subsection{Composición química inicial}

La composición química inicial de la muestra de colas se presenta en la tabla 1.

Tabla 1: Composición química inicial de las colas. 


\begin{tabular}{c|c|c|c|c|c|c|c|c}
\hline Elementos & $\mathrm{Ni}$ & $\mathrm{Co}$ & $\mathrm{Fe}$ & $\mathrm{Mg}$ & $\mathrm{Mn}$ & $\mathrm{Al}$ & $\mathrm{Cr}$ & $\mathrm{SiO}_{2}$ \\
\hline Contenido, \% & 0,37 & 0,06 & 43,67 & 3,77 & 0,69 & 5,41 & 2,72 & 9,89 \\
\hline
\end{tabular}

Se observa que los elementos que aparecen en mayor cantidad son hierro, sílice, aluminio, magnesio y cromo, en menor cuantía manganeso, níquel y cobalto. Estos resultados son similares a los obtenidos por diversos investigadores (Rojas \& Turro, 2003; Hernández, 2011; García, 2011 y Loyola, 2012. En tal sentido se puede considerar las colas como un mineral de hierro que contiene más del $40 \%$ del elemento; por tanto, es una reserva potencial para los productores de acero a nivel mundial. De igual manera las colas contienen cantidades apreciables de níquel y cobalto, dado por la ineficiencia tecnológica de la planta que procesan los minerales; lo que brinda una posible fuente de estudio para su futura recuperación.

\subsection{Análisis estadístico}

Se realizó la modelación estadística matemática en los productos de mayor concentración para dos elementos mediante el software Statgraphics 5.1 Plus. Mediante el modelo se puede predecir la concentración del elemento a partir de las variables granulometria y densidad.

La figura 3 muestra los efectos principales para el níquel en el reboso. Al aumentar la granulometría de 0,02 a 0,08 mm aumentó el contenido de níquel de 0,35 a 0,98\%, con un crecimiento de $0,63 \%$. La densidad describe un comportamiento prácticamente lineal, aunque hubo una disminución en la concentración de 0,64 a 0,59 \% al aumentar la densidad.

La figura 4 muestra la superficie de respuesta para el níquel, la concentración del elemento aumenta proporcionalmente al ascender la granulometria, comportamiento similar descrito en la figura 3; en tanto, el cambio de la densidad no afecta la concentración de níquel. Mediante la superficie de respuesta se determina el modelo matemático que mejor se ajusta a los datos obtenidos.

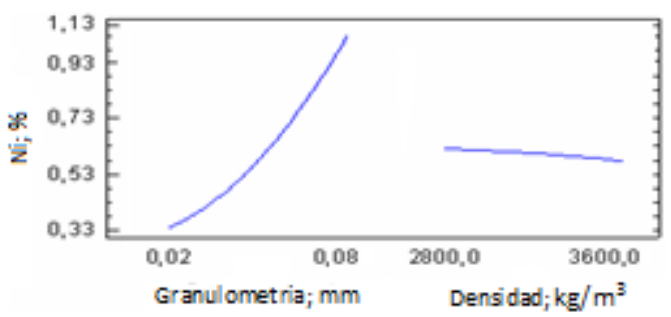

Figura 3: Efectos principales.

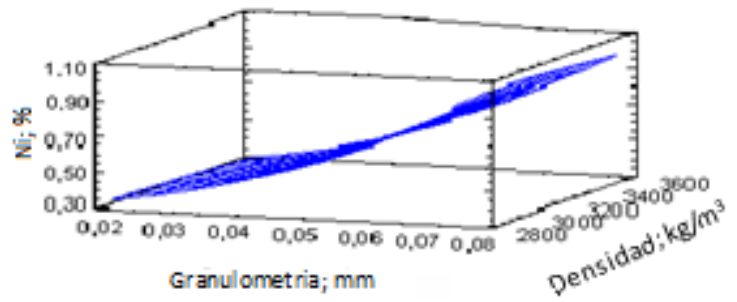

Figura 4: Superficie de respuesta.

La ecuación del modelo de regresión ajustada para el níquel en el reboso es:

$$
N i=0,07+1,3 \cdot G+0,00016875 \cdot D+116,667 \cdot G^{2}-0,0005 \cdot G \cdot D-3,125 E-8 \cdot D^{2}
$$

La figura 5 muestra los efectos principales para el hierro en el reboso. Al aumentar la granulometría de 0,02 a 0,08 mm, hubo un decrecimiento en el contenido del elemento de 49,80 a $38,20 \%$, con una disminución de $23,2 \%$.

Al aumentar la densidad de 2800 a $3600 \mathrm{~kg} / \mathrm{m}^{3}$ hubo un aumento en la concentración de hierro de 44,50 a 44,97\%, con un aumento de 1,05\%. La figura 6 muestra la superficie de respuesta 
estimada, donde se observa un comportamiento prácticamente planar de la composición de hierro con relación a la densidad y granulometría.

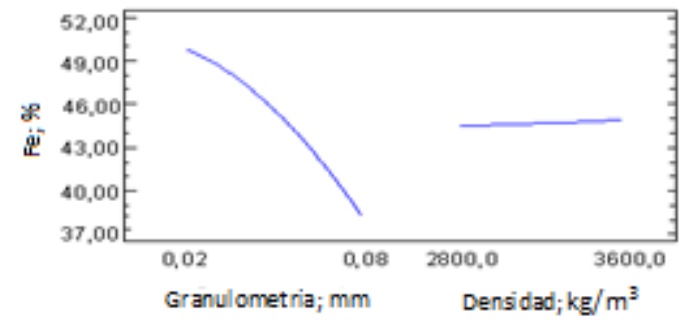

Figura 5: Efectos principales.

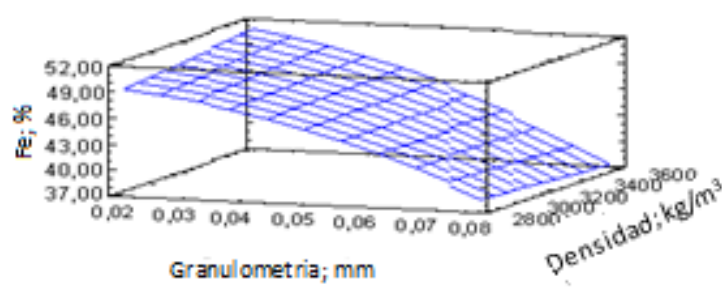

Figura 6: Superficie de respuesta.

La ecuación del modelo de regresión ajustada para el hierro en el reboso es:

$F e=39,725+166,0 \cdot G+0,0038125 \cdot D-1916,67 \cdot G^{2}-0,055625 \cdot G \cdot D-7,8125 E-8 \cdot D^{2}$

\subsection{Comportamiento denso-granulométrico de las colas}

De los resultados presentados en la tabla 2, se aprecia que las partículas menores de 0,04 $\mathrm{mm}$ representan más del $50 \%$ de la masa total del sólido, debido a que fueron separadas cuando la velocidad del flujo ascendente las arrastra, teniendo en consideración la densidad; corroborando lo planteado por Turro (2002) y Loyola (2012), cuando en la separación por tamices obtuvieron valores semejantes.

Tabla 2: Composición denso-granulométrica de las colas.

\begin{tabular}{|c|c|c|c|c|c|c|}
\hline $\begin{array}{c}\text { Tamaño de } \\
\text { partícula, } \\
\text { mm }\end{array}$ & $\begin{array}{c}\text { Densidad, } \\
\mathrm{kg} / \mathrm{m}^{3}\end{array}$ & $\begin{array}{c}\text { Masa de } \\
\text { análisis, } \\
\text { kg }\end{array}$ & $\begin{array}{c}\text { Masa } \\
\text { reboso, } \\
\text { kg }\end{array}$ & $\begin{array}{c}\text { Masa } \\
\text { arenas, } \\
\text { kg }\end{array}$ & $\begin{array}{c}\text { Masa análisis } \\
\text { químico, } \\
\text { kg }\end{array}$ & $\begin{array}{c}\text { Salida } \\
\text { acumulativa, } \\
\%\end{array}$ \\
\hline \multirow{3}{*}{0,02} & 2800 & 0,2500 & 0,0294 & 0,2206 & 0,0040 & \multirow{3}{*}{27,64} \\
\hline & 3200 & 0,2166 & 0,0204 & 0,1962 & 0,0040 & \\
\hline & 3600 & 0,1922 & 0,0193 & 0,1729 & 0,0040 & \\
\hline \multirow{3}{*}{0,04} & 2800 & 0,1689 & 0,0272 & 0,1417 & 0,0040 & \multirow{3}{*}{51,64} \\
\hline & 3200 & 0,1377 & 0,0169 & 0,1208 & 0,0040 & \\
\hline & 3600 & 0,1168 & 0,0159 & 0,1009 & 0,0040 & \\
\hline \multirow{3}{*}{0,06} & 2800 & 0,0969 & 0,0166 & 0,0803 & 0,0040 & \multirow{3}{*}{64,60} \\
\hline & 3200 & 0,0763 & 0,0082 & 0,0681 & 0,0040 & \\
\hline & 3600 & 0,0641 & 0,0076 & 0,0565 & 0,0040 & \\
\hline 0,08 & 2800 & 0,0525 & 0,0103 & 0,0422 & 0,0040 & 73,20 \\
\hline
\end{tabular}




\begin{tabular}{l|l|l|l|l|l|l}
\hline & 3200 & 0,0382 & 0,0062 & 0,0032 & 0,0040 & \\
\cline { 2 - 5 } & 3600 & 0,0028 & 0,0005 & 0,0023 & 0,0040 & \\
\hline
\end{tabular}

La salida acumulativa de las partículas menores de 0,08 mm del reboso representa el 73,20 $\%$ de todo el material, lo que evidencia una distribución de partículas finas en todo el espectro granulométrico; tal aspecto corrobora los resultados alcanzados por Turro (2002); Hernández (2011); García (2011) y Loyola (2012).

3.4 Comportamiento químico de los elementos en la concentración densogranulométrica

El comportamiento químico de los elementos se analizó a través de la composición química obtenida en cada uno de los resultados. La figura 7 muestra el comportamiento de níquel, magnesio y sílice en los dos productos, donde los tres metales tienen una tendencia a concentrarse en el reboso de las fracciones 0,06 y $0,08 \mathrm{~mm}$; debido a que la sílice y magnesio son elementos asociados a minerales de baja densidad y, por esa condición, son arrastrados por el flujo ascendente, o sea, ofrecen poca resistencia. A medida que se incrementa el tamaño de las partículas, aumentan su concentración; esto se debe a que los tres elementos están presentes en la serpentina, la cual es de poca molibilidad; por tanto, se concentra en las fracciones más gruesa. Así, se corrobora lo planteado por Coello (2008) y Loyola (2012); además, estos metales disminuyen su concentración a medida que aumenta la densidad en cada fracción, debido a lo explicado anteriormente respecto a la asociación con minerales de baja densidad. En las arenas los elementos mantuvieron un comportamiento semejante y se debe a la baja densidad de sílice y magnesio en el producto y a la asociación del níquel con ellos, prácticamente no hay concentración.

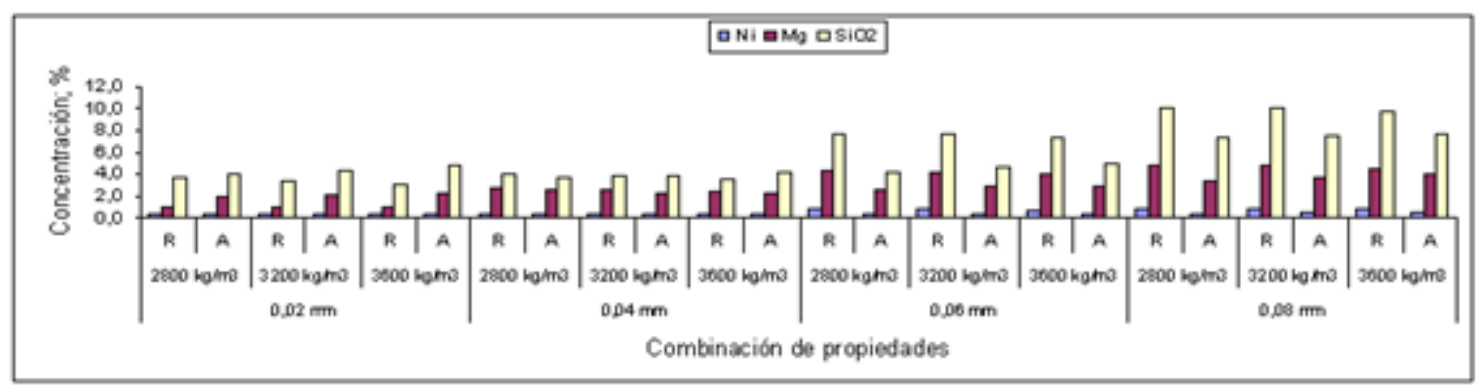

Figura 7: Comportamiento de níquel, magnesio y sílice.

A partir del tamaño de partícula 0,06 mm del reboso se observó una notable tendencia a la concentración de los metales, lo que se mostró más intenso para la clase más gruesa. Esta afirmación se debe a que, a partir de ese tamaño de partícula, básicamente, hay un mayor predominio de la serpentina.

El níquel, en la combinación $0,08 \mathrm{~mm}$ y $2800 \mathrm{~kg} / \mathrm{m}^{3}$, tuvo concentración de $1,0 \%$ en el reboso y disminuye a $0,96 \%$ al aumentar la densidad a $3600 \mathrm{~kg} / \mathrm{m}^{3}$. En las fracciones 0,06 y 0,08 $\mathrm{mm}$ de las arenas, a mayor densidad aumenta la concentración de níquel, para 0,06 mm de 0,39 a 0,40\%; en tanto; para la fracción gruesa de 0,45 a $0,49 \%$, lo que se debe a la alta densidad que posee el elemento, así como a su asociación con las fases mineralógicas del hierro, fundamentalmente con la goethita (Rojas, 2001). 
El magnesio, en la combinación de $0,08 \mathrm{~mm}$ y $2800 \mathrm{~kg} / \mathrm{m}^{3}$, tuvo concentración de $4,8 \%$ en el reboso; al aumentar la densidad hasta $3600 \mathrm{~kg} / \mathrm{m}^{3}$ disminuye la concentración a 4,56 \%. En las fracciones 0,06 y $0,08 \mathrm{~mm}$ de las arenas, a mayor densidad aumenta la concentración, para el primer caso de 2,73 a 3,00 \%; en tanto, para la fracción gruesa de 3,50 a 3,98 \%, lo que se debe fundamentalmente a la gruesa granulometría que presenta el elemento.

La sílice, en la combinación de 0,08 mm y $2800 \mathrm{~kg} / \mathrm{m}^{3}$, alcanza concentración de 9,98 \% en el reboso y disminuye a $9,7 \%$ al aumentar la densidad a $3600 \mathrm{~kg} / \mathrm{m}^{3}$. Para la sílice se observa un comportamiento similar al níquel y magnesio; en fracciones mayores de las arenas, a mayor densidad crece la concentración, para $0,06 \mathrm{~mm}$ de 4,30 a 4,94 \%, en tanto; para la fracción gruesa de 7,30 a 7,65 \%, lo que se debe fundamentalmente a la formación de compuestos amorfos (Chang, 1999).

El hierro manifestó un comportamiento contrario al níquel, magnesio y sílice. En la figura 8 se observa mayor predominio en las fracciones 0,02 y $0,04 \mathrm{~mm}$; ya que el elemento tiene mayor influencia en las partículas finas. Al combinar la granulometría $0,02 \mathrm{~mm}$ con las tres densidades en forma ascendente, el hierro incrementa su concentración en el reboso de 49,0 a 50,4 \%. Se confirma lo planteado por Turro (2002); Loyola (2012) y otros investigadores, quienes afirman que el hierro está mayormente distribuido en las fracciones más finas; se atribuye además a lo planteado por Rojas, 2001 respecto a que el elemento está presente en la limonita.

La elevada concentración del elemento se puede atribuir a que en los minerales que dan origen a las colas, el hierro está en su mayor concentración en la limonita (grupo de mena fundamental); principalmente como fase de goethita que puede llegar hasta un 69 \% (Laverov, 1985; Ostroumov et al. 1987; Rojas, 2001).

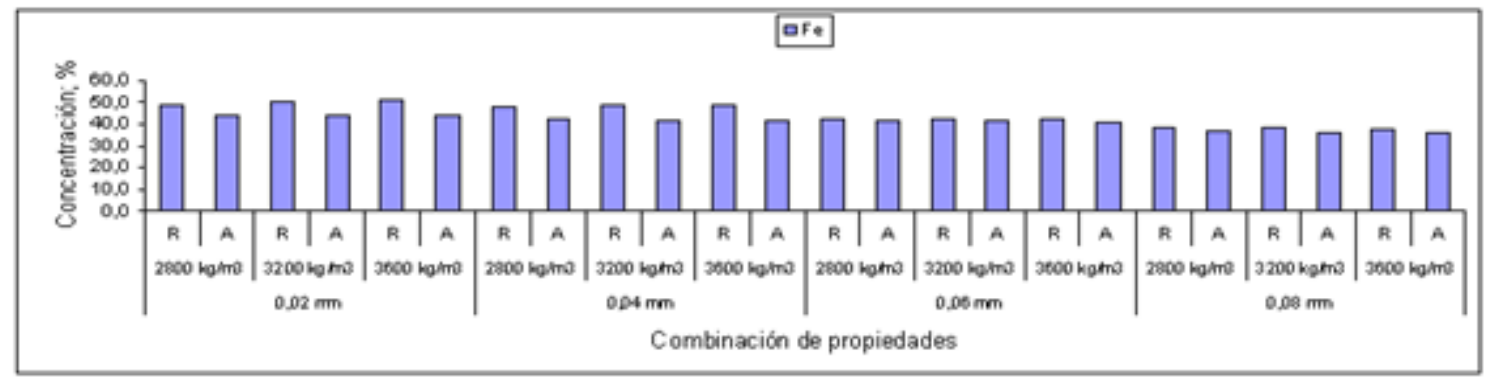

Figura 8: Comportamiento del hierro.

El cobalto y manganeso (figura 9) tienden a concentrarse en la fracción 0,04 mm, en las arenas; el primero aumenta su contenido de 0,06 a 0,07 \% al aumentar la densidad a $3600 \mathrm{~kg} / \mathrm{m}^{3}$. El segundo alcanza concentración de $0,83 \%$ a la densidad de $2800 \mathrm{~kg} / \mathrm{m}^{3}$, el cual disminuye a 0,82 $\%$ al aumentar la densidad a $3600 \mathrm{~kg} / \mathrm{m}^{3}$. En la fracción $0,04 \mathrm{~mm}$ los elementos mantienen un comportamiento lineal (estadístico $\mathrm{R}^{2}$ igual a 70,6 y 83,9 \% respectivamente) y se debe fundamentalmente a que las asbolanas (fase principal de manganeso) es la principal portadora de cobalto (Barros de Oliveira et al. 2001). 


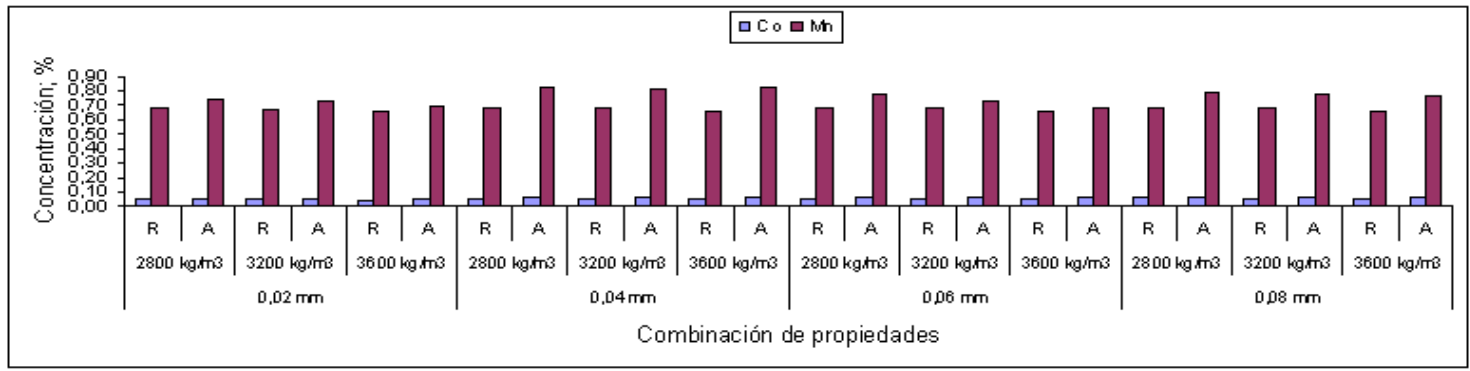

Figura 9: Comportamiento del cobalto y manganeso.

El cromo (figura 10) disminuye su concentración al aumentar la densidad en el reboso. En la fracción 0,04 $\mathrm{mm}$ de las arenas al aumentar la densidad aumenta su concentración de 2,75 a 2,83 $\%$, debido a que el metal es de elevada densidad, además Rojas (2003) plantea la asociación del cromo con las fases mineralógicas de hierro, específicamente con las cromo espinelas.

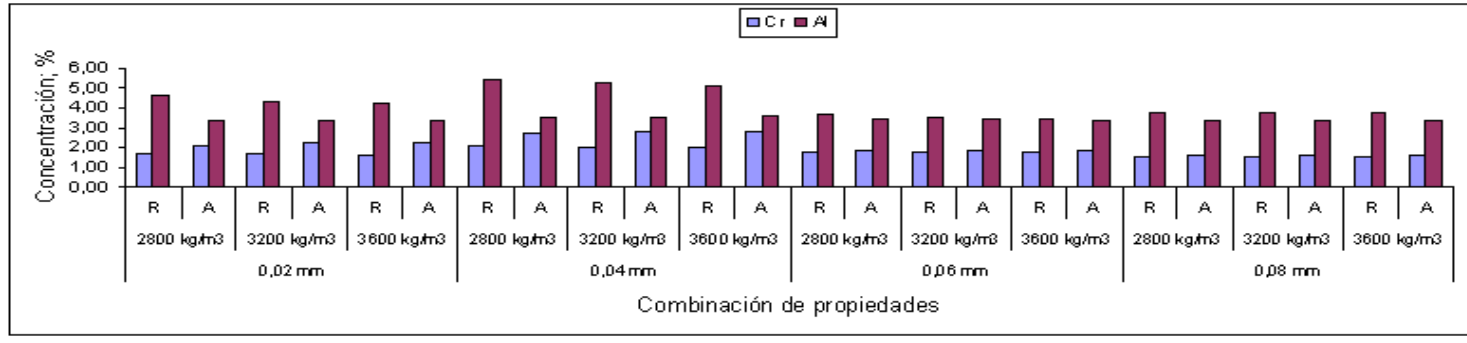

Figura 10: Comportamiento del cromo y aluminio.

El aluminio, en el reboso con la combinación de $0,04 \mathrm{~mm}$ y $2800 \mathrm{~kg} / \mathrm{m}^{3}$ obtiene su mayor concentración de 5,37\%. El alto contenido se puede atribuir a la sustitución del hierro por el aluminio en la red cristalina de la goethita, fenómeno muy común entre los óxidos de hierro en un ambiente oxidante y en presencia de silicatos e hidróxidos de aluminio (Schwertmann et al. 2000). En las arenas existe un comportamiento homogéneo, las concentraciones se debe a la asociación con las cromo espinela.

\section{CONCLUSIONES}

La Empresa Ernesto Che Guevara genera gran volumen de residuos con concentraciones importantes de metales de interés por su baja eficiencia metalúrgica, los que pueden ser concentrados en diferentes combinaciones de densidad y granulometría con el propósito de recuperarlos, y con esto reducir el impacto ambiental. En el reboso, en las fracciones más finas se pueden recuperar el hierro y aluminio, a la mayor y menor densidad respectivamente. En la fracción gruesa a la menor densidad se recupera el níquel, magnesio y silicio. En las fracciones más finas y a menor densidad de las arenas se recupera el manganeso, mientras a la mayor densidad lo hacen el cobalto y cromo. 


\section{REFERENCIAS}

Ariza, S., Salazar, Y., \& Capote, N. B. (2004). Lixiviación química de colas producto del proceso carbonato amoniacal con ácido orgánico. Revista Tecnología Química, 24-36.

Barros de Oliveira, S. M., Moya, C. S., \& Enzweiler, J. (2001). Ochereous laterite: a nickel ore from Punta Gorda. Journal of South Amarican Earth Sciences, 307-317.

Chang, A. (1999). Una visión sobre las estratégias de eficiencia metalúrgica en la tecnología carbonato-amoniacal. Revista Geología y Minería, 76-82.

Coello, A., Menéndez, J. M., \& Laborde, R. (2008). Gridability of lateritic nickel ore in Cuba. Journal Powder Technology, 113-115.

Col, L., \& García, L. (1984). Investigaciones complementarias sobre el beneficio de las colas de Nicaro a escala de laboratorio y de planta piloto. Nicaro.

Coulson, J. M., \& Richardson, J. F. (1979). Ingeniería Química. Barcelona: Reverté.

Del Toro, A. (2003). Consideraciones sobre la posible recuperación del cobalto contenido en las colas de la tecnología carbonato amoniacal. (Tesis de pregrado). Instituto Superior Minero Metalúrgico, Moa.

García, D. (1988). Estudio preliminar de la obtención del concentrado de cromo a partir de las colas de Nicaro. (Tesis de pregrado). Instituto Superior Minero Metalúrgico, Moa.

García, E. (2011). Extracción de cobalto por lixiviación ácida de los residuales sólidos de la tecnología carbonato amoniacal. (Tesis de maestría). Instituto Superior Minero Metalúrgico, Moa.

Goodell, T. (1980). Un dispositivo de elutriación de partículas, más particularmente para separar coque de desecho de descarga, junto con un método correspondiente. EEUU. Patente № 123.456.

Hernández, C. M. (2011). Recuperación de Níquel, Cobalto y Hierro a partir del residuo sólido de la empresa "Ernesto Che Guevara". (Tesis de maestría). Instituto Superior Minero Metalúrgico, Moa.

Herrera, V. (1961). Comentarios sobre el informe del Instituto Mejanobr sobre el proyecto para la utilización de las colas de Nicaro como mineral de hierro. Nicaro.

Kowalski, T. (1953). Proposed flow sheet for metals recovery from Nicaro tailings. Nicaro.

Laverov, N. P. (1985). Yacimientos minerales metálicos de Cuba. URSS: Nauka.

Loyola, O. (2012). Comportamiento de las colas de la empresa "Comandante Ernesto Che Guevara" ante la variación de la intensidad del campo magnético. (Tesis de maestría). Instituto Superior Minero Metalúrgico, Moa. 
Loyola, O., Beyris, P., Palacios, A., \& Correa, Y. (2014). Comportamiento de las colas del proceso Caron (Moa) ante la intensidad de un campo magnético. Revista Geología y Minería, 104116.

Medrano, A. (2007). Lixiviación Ácida de los Residuales Sólidos de la tecnología Carbonato amoniacal. (Tesis de pregrado). Instituto Superior Minero Metalúrgico, Moa.

Mitrofanov, S. I. (1982). Investigación de la capacidad de enriquecimieno de los minerales. Moscú: MIR.

Osorio, C. A., Marin, J. M., Pelaez, R. J., \& Restrepo, V. G. (2009). Evaluación de un sistema de clasificación hidráulica para separación diferencial de mezclas sólidas. Revista Ingeniería e Investigación, 36-41.

Ostroumov, M. N., Blanco, J., \& Rojas, A. (1987). Caracterización mineralógica de las lateritas del sector Atlantic de la mina Moa. Revista Geología y Minería, 3-20.

Palmero, A. (2011). Comportamiento de las colas de la empresa "Comandante Ernesto Che Guevara" ante la intensidad de un campo magnético. (Tesis de pregrado). Instituto Superior Minero Metalúrgico, Moa.

Peña, E. (1982). Estudio de sedimentación fraccional. (Tesis de pregrado). Instituto Superior Minero Metalúrgico, Moa.

Peña, J. G. (1986). Estudio del esquema de beneficio de las colas de Nicaro. (Tesis de pregrado). Instituto Superior Minero Metalúrgico, Moa.

Ponce, N. (1979). Mineralogía y composición sustancial del yacimiento artificial Colas de Nicaro. Nicaro.

Ponce, N., \& Altarriba, I. C. (1986). Composición sustancial del yacimiento artificial Colas de Moa. Revista Tecnológica-Serie Geológica, 66-75.

Resumen Taller de Colas. (2005). Análisis de información científico técnica sobre el procesamiento de las colas de mineral. Moa.

Rojas, A. (2001). Evidencias a favor de la goethita es la principal portadora de níquel en los horizontes lateríticos de las cortezas ferroniquelíferas. Revista Geología y Minería, 21-31.

Rojas, A. (2003). Características mineralógicas de los residuales no lixiviados del proceso Caron, en Moa, Holguín, Cuba. Revista Geología y Minería, 23-34.

Rojas, A., \& Turro, A. (2003). Composición mineralógica de las colas del proceso CARON en Moa, Holguín, Cuba. Revista Geología y Minería, 21-28.

Schwertmann, U., Friedl, J., Stanjek, H., \& Schulze, D. (2000). The effect of Al on Fe oxides; XIX, Formation of $\mathrm{Al}$ - substituted hematite from ferrihydrite at 25 degrees $\mathrm{C}$ and $\mathrm{pH} 4$ to 7 . $\mathrm{G}$. Journal Clays and Clays Minerals, 159-172.

Svarovsky, L. (2000). Solid-Liquid Separation. Praga: Butterworth-Heinemann. 
Turro, A. (2002). Estudio del Hidrotransporte de las Colas en el Proceso Carbonato Amoniacal. (Tesis doctoral). Instituto Superior Minero Metalúrgico, Moa. 